Review

\title{
Silver-based wound dressings: current issues and future developments for treating bacterial infections
}

\author{
Haidari H, Garg S, Vasilev K, Kopecki Z and Cowin AJ
}

Keywords wound infection, anti-biofilm, chronic wounds, silver dressings, antibacterial dressing

For referencing Haidari $\mathrm{H}$ et al. Silver-based wound dressings: current issues and future developments for treating bacterial infections. Wound Practice and Research 2020; 28(4):173-180.

DOI https://doi.org/10.33235/wpr.28.4.173-180

\section{Hanif Haidari}

BMedSc (Hons)

PhD Candidate, UniSA Clinical \& Health Sciences,

University of South Australia, Adelaide, SA

Australia

Future Industries Institute, University of South

Australia, Mawson Lakes, SA, Australia

\section{Sanjay Garg}

$\mathrm{PhD}$

Professor of Pharmaceutical Science, UniSA

Clinical \& Health Sciences, University of South

Australia, Adelaide, SA, Australia

\section{Krasimir Vasilev}

PhD

Professor, Academic Unit of STEM, University of

South Australia, Mawson Lakes, SA, Australia

Future Industries Institute, University of South

Australia, Mawson Lakes, SA, Australia

\section{Zlatko Kopecki}

$\mathrm{PhD}$

Research Fellow, Future Industries Institute,

University of South Australia, Mawson Lakes, SA,

Australia

UniSA Clinical \& Health Sciences, University of

South Australia, Adelaide, SA, Australia

\section{Allison J Cowin*}

$\mathrm{PhD}$

Professor, Future Industries Institute, University of

South Australia, Mawson Lakes, SA, Australia

UniSA Clinical \& Health Sciences, University of

South Australia, Adelaide, SA, Australia

Email Allison.Cowin@unisa.edu.au

${ }^{*}$ Corresponding author

\section{Abstract}

The rapid and continuing emergence of antimicrobial resistance is challenging our ability to effectively fight bacterial infections. This challenge is particularly felt in clinical settings where patients experience severely infected chronic wounds. Despite the increased development and reliance on silver-based dressings, the evidence underpinning their clinical efficacy is inconclusive and there remain concerns and confusion about their use.

In this review, we describe the rationale for the current use of silver and silver-based wound dressings and highlight the progress and challenges associated with developing more advanced nanoparticle-based dressings for the safe and effective delivery of silver. Specifically, we describe the development of silver nanoparticles (AgNPs) with unique characteristics that render them applicable for controlling bacterial infections while simultaneously promoting wound healing through mechanisms including the dampening of inflammation and promotion of wound re-epithelialisation. Finally, we describe the latest progress in the development of different types of hydrogels for the therapeutic delivery of AgNPs to infected wounds. The possibility of designing and establishing multimodal silver therapies for the safe and effective treatment of infected wounds would be of great benefit for wound care management and improved patient outcomes.

\section{Introduction}

Wound healing is a multi-step, highly coordinated and regulated process that occurs in overlapping phases of haemostasis, inflammation, proliferation and remodelling1. Aimed at rapidly restoring tissue integrity and functions, these processes are highly regulated in the coordination of multiple cell types, including keratinocytes, fibroblasts, endothelial and immune cells, as well as numerous growth factors ${ }^{2}$. However, open wounds are highly susceptible to bacterial colonisation which is described as the presence 
of multiplying bacterial populations in close proximity with no associated clinical signs and symptoms. A heavier colonisation load, also known as critical colonisation, may predispose an individual to wound infection ${ }^{3,4}$. Wound infection is described as the invasion of proliferating bacterial cells which are present at critically high levels $-10^{5}$ colony forming units (CFU) of bacteria per gram of tissue - where the balance between virulence and host immunity tilts in favour of microorganisms ${ }^{5,6}$. In this stage, infection can trigger a cascade of host responses, including presentation of active disease symptoms - tissue inflammation, pus and exudate - which requires antibacterial treatment ${ }^{7}$. Furthermore, excessive accumulation of bacterial cells can result in bacteria forming a collective community cluster protected by self-produced exopolysaccharides (EPS) known as bacterial biofilms ${ }^{8}$. Consequently, wound infection raises critical healthcare concerns as it is one of the main causative factors for delayed wound healing which can result in serious detrimental effects ${ }^{9}$. Our inability to effectively clear bacteria at the early stages of colonisation increases the risk of severe infection and aids the production of biofilms ${ }^{10}$.

In Australia, more than $10 \%$ of patients will acquire a chronic wound infection, adding to the significant healthcare burden of $\$ 2.85$ billion annually that is attributed to wound care ${ }^{11-13}$. Additionally, biofilms are reported to be associated with $80 \%$ of chronic wounds and significantly contribute to their impaired healing ${ }^{14}$. Once established, biofilms are highly resistant and are recognised as a major contributor to the development of bacterial resistance to antibiotics ${ }^{15}$. Biofilms prevent antibiotic penetration into infected wounds and often result in treatment failure ${ }^{16}$.

In response to the increased incidence of multidrug-resistant (MDR) bacteria and the lack of new antibiotics in research and development, research focus has again shifted back to investigating the antibacterial capabilities of silver; this, however, is now with a stronger emphasis on new therapeutic modalities that can simultaneously promote tissue regeneration and control bacterial infection. The antimicrobial activity of silver has been used for hundreds of years in wound care with broad spectrum activity observed against a range of bacteria ${ }^{17,18}$. However, certain limitations, including its possible adverse effects on healthy skin tissue, have restricted its widespread use and have prompted research towards optimising the therapeutic benefits of silver while minimising the potential side effects for the treatment of wounds. These developments will be discussed and summarised in this review.

\section{The use of silver in wound healing}

Silver as an antimicrobial agent

Silver is a transition metal known to have many useful biological properties and is well-recognised for its broad spectrum antibacterial effects against both gram-negative and gram-positive bacteria. Silver in its metallic state $\left(\mathrm{Ag}^{\circ}\right)$ is inert, while silver in its monoatomic state $\left(\mathrm{Ag}^{+}\right)$is biologically active and poses strong bactericidal activity ${ }^{19}$. Oxidation of silver atoms readily occurs in the presence of molecular oxygen $\left(\mathrm{O}_{2}\right)$, leading to biologically active silver ions. The medical application of silver ions has been well documented for centuries and it has been employed as the first-choice treatment for many ulcerations and infectious diseases including venereal disease, bone perianal abscess, eye drops and burn wounds ${ }^{20,21}$. The discovery of antibiotics in the early 20th century led to a decline in the use of silver as an antimicrobial agent. However, the emergence of antibiotic resistance in the early 1960 s led to a renewed interest in the use of silver products, including concentration-dependent $(0.5 \%$ and $1 \%)$ silver nitrate $\left(\mathrm{AgNO}_{3}\right)$ for the treatment of burns, $1 \%$ topical cream formulations, for example silver sulfadiazine (Ag-SD), and many other forms of advanced silver dressings ${ }^{22,23}$. Over the last 2 decades, this resurgent interest in silver has led to large-scale productions of numerous silver-containing dressings impregnated with silver salt or metallic silver, a selection of which is included in Table $1^{5,19}$.

The difference in current silver wound dressings is largely based on both silver composition and the amount of active silver that is released into the wound. Mostly, the reported dressings are manufactured with the simple impregnation of silver nitrate into a dressing material that is susceptible to unregulated fast release, resulting in short-term activity and the need for frequent dressing changes ${ }^{24}$. Additionally, traditional silver dressings are susceptible to rapid inactivation by wound fluid, resulting in chemical complexes that can prevent their antibacterial activity ${ }^{25}$. To compensate for the potential loss of activity, the amount of silver in the wound dressings is excessively increased to replenish silver ions ${ }^{26}$.

However, excessive accumulation of silver into wounds has been shown to inhibit skin cell proliferation and impair wound healing ${ }^{27,28}$ and in vitro and in vivo studies have shown that application of silver dressings can increase host tissue toxicity and that care must be taken for its widespread usage ${ }^{29,30}$. Consequently, advances in the field of nanotechnology have enabled the possibility of developing silver in the form of nanoparticles (NPs) to deliver significantly smaller amounts of silver into the wounds, hence improving safety without compromising its excellent antibacterial properties ${ }^{31,32}$. Progress in this field is reflected by the increased number of patents developing silver for medicinal applications over the last decade (2007-2017), emphasising the high potential for industrial and healthcare applications ${ }^{33}$.

\section{Silver nanoparticles}

NPs are materials engineered with at least one dimension within the nanoscale range $(1-100 \mathrm{~nm})^{34}$. A one-nanometre particle represents $10^{-9}$ metres in length which brings a significant difference in dimension and functionality compared to bulk counterparts. The major advantage of NPs is the versatility and tunability of their physicochemical properties depending on their application to medical purposes, including 
controlled drug delivery, improved efficacy and enhanced transport across biological barriers ${ }^{35}$. The continuing interest in silver nanoparticles (AgNPs) is related to the bactericidal efficacy of AgNPs at a much lower concentration than silver ions, as well as decreased toxicity to mammalian cells and other unique properties enabling optimisation of surface functionality ${ }^{36}$. AgNPs provide a sustained source of silver ions as the gradual oxidation of silver atoms releases biologically-active silver ions, providing a constant flux of silver that can target key bacterial pathogen structures ${ }^{37}$. In contrast to conventional silver nitrate, the combined action of a continuous supply of silver ions and the optimal physicochemical properties of AgNPs pose a substantial threat to bacterial survival when exposed to the treatment. Additionally, the versatile and dynamic properties of AgNPs provide a greater degree of flexibility in manipulating key physicochemical properties, including structure, size, surface functionalisation and biocompatibility, to achieve desirable antimicrobial benefits ${ }^{38}$. In particular, the large surface area of AgNPs allows more prominent numbers of active sites for interaction with biological species and makes AgNPs a better material than usual bulk materials ${ }^{39}$. The knowledge of how AgNPs and bacterial cells interact has greatly increased over the past decade and, as a result, several possible mechanisms of bactericidal action have been proposed; these are described below.

Table 1. A selection of commercial silver wound dressings

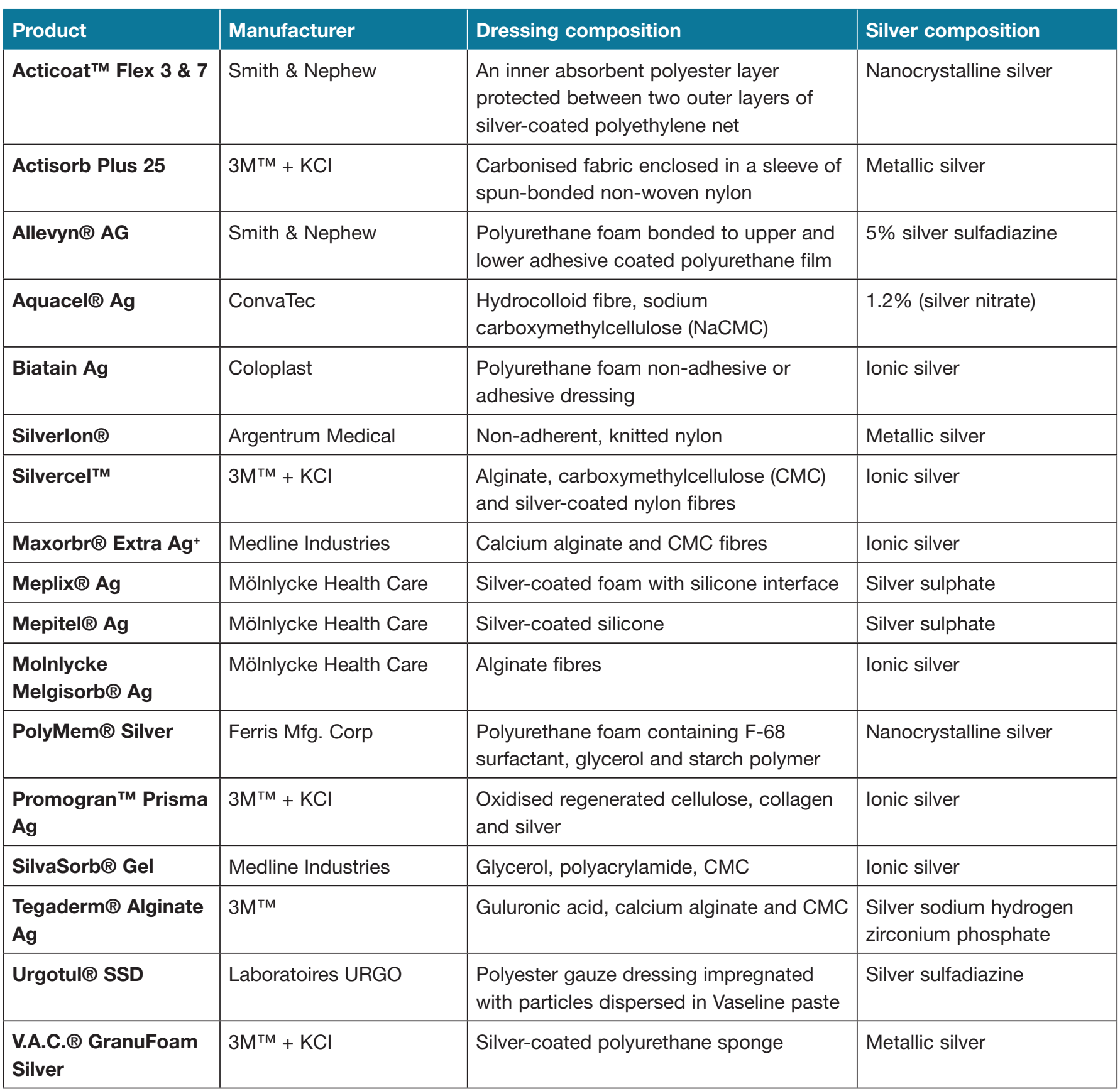


Antibacterial mechanisms of AgNPs

The continuous leaching of silver ions from AgNPs is effective against a broad spectrum of bacteria, including both gram-negative and gram-positive bacteria ${ }^{26}$. While it is clear that silver ions are highly reactive and possess a high affinity for organic amines, phosphates and, most notably, thiols present in DNA, proteins and cell membranes, it remains unclear whether the NPs themselves contribute to bacterial toxicity ${ }^{40,41}$.

There are three currently accepted mechanisms for the antibacterial action of AgNPs. Firstly, AgNPs can physically interact with a bacterial cell membrane, causing surface corrugation and hence an increase in membrane permeability, allowing AgNPs to penetrate intracellularly ${ }^{42}$. Secondly, AgNPs are responsible for attacking respiratory proteins and impairing the respiratory chain, hence hindering the production of energy and causing interference with mitochondria to generate reactive oxygen species (ROS) ${ }^{43}$. Finally, AgNPs can penetrate inside the bacterial cells which allows interaction of AgNPs with cellular structures and biomolecules, including lipids, proteins and DNA, leading to disruption of key cellular events and bacterial functioning ${ }^{44}$. These important dynamic processes lead to severe impairment of essential cellular functions, ultimately leading to bacterial cell necrosis and death $^{36}$.

AgNPs are shown to be effective against a wide range of microorganisms, including bacteria, fungi and anaerobes, and the susceptibility differences are pathogen-specific based on the type of organism and membrane structures ${ }^{18}$. However, AgNPs mechanisms of action have largely been shown to be similar across different pathogens in targeting multiple key membrane structures to cause pathogen cell death. For example, Kim et al. report that 13.4nm AgNPs showed the minimum inhibitory concentration (MIC) against E. coli to be $6.6 \mathrm{nM}$ compared to $33 \mathrm{nM}$ for $S$. aureus ${ }^{45}$. The differences depend on the peptidoglycan layer where gramnegative bacteria (i.e. E. coli and $P$. aeruginosa) are more susceptible than gram-positive bacteria (i.e. S. aureus and S. epidermidis). Recent studies have also proposed that the simultaneous interaction of AgNPs on multiple targets in bacterial cells makes it difficult for bacteria to develop silver resistance, a key advantage against current antibiotics ${ }^{36,46}$. A more detailed review of the antibacterial mechanism of AgNPs is reported elsewhere ${ }^{19,47}$.

Physicochemical properties of AgNPs enable high antibacterial activity

AgNPs exhibit particle-specific activity, where the pathways involved in the antibacterial activity are highly dependent on the physicochemical properties of NPs. The physical and chemical properties of AgNPs' structure - size and surface structure - are important for their toxicology ${ }^{48}$. In particular, the size of the NPs is the crucial factor that influences their intrinsic antibacterial properties ${ }^{49}$. The influence of particle size has been demonstrated in cell toxicity, tissue distribution, dermal penetration and cellular uptake ${ }^{41}$. Morones et al. demonstrate the size dependence activity of AgNPs (1-100nm) against a range of bacteria, namely $E$. coli, $V$. cholera, S. typhus and $P$. aeruginosa ${ }^{50}$. AgNPs in the range of $1-10 \mathrm{~nm}$ showed significantly higher affinity in the attachment to the bacterial surface compared to larger sized AgNPs. Similarly, Agnihotri et al. studied the 5-100nm size of AgNPs and observed that the smallest AgNPs $(5 \mathrm{~nm})$ showed the fastest and most efficient bacterial killing ${ }^{51}$. Additionally, many studies reveal that AgNPs smaller than 10nm provide favourable antibacterial properties ${ }^{49,52}$ and have a greater tendency to penetrate bacteria compared to large $\mathrm{AgNPs}^{53}$. Therefore, the unique size of AgNPs is particularly relevant in response to treating bacterial biofilms.

AgNPs have also been shown to physically interact with the biofilm matrix to deteriorate and disassemble the biofilm integrity as a route to access the bacterial cells ${ }^{54}$. The effective removal of biofilm depends on the diffusion capacity of the antimicrobial agent, where smaller NPs bring significant advantages ${ }^{40}$. Hence, small-sized AgNPs are expected to readily diffuse through the biofilms to release a high concentration of silver ions, eradicating the bacterial biofilms ${ }^{54}$. For example, Choi et al. show that 20nm AgNPs can penetrate up to $40 \mu \mathrm{m}$ in E. coli biofilm within the first hour, which is a significant advancement on the penetration of both antibiotics and silver nitrate ${ }^{55}$. In the context of chronic wounds, AgNPs provide a significant improvement to current silver dressings which are limited to killing only planktonic cells due to the nature of silver delivery and poor penetration of bacterial biofilms associated with chronic wounds.

In addition to the size, the surface charge of AgNPs is another important factor that determines its biological effectiveness. Most microbes display an overall negative surface charge and are expected to provide a higher affinity for positively charged AgNPs due to electrostatic interaction. Recent studies have shown to enhance the retention of AgNPs on the bacterial surface via electrostatic mediated interaction ${ }^{56,57}$. A recent study by El Badaway et al. demonstrates that the surface charge of AgNPs is the most important factor that governs the AgNPs' toxicity to microbes ${ }^{58}$. Here, negatively charged, citrate stabilised AgNPs were the least toxic, whereas positively charged AgNPs $(+38 \mathrm{mV})$ showed the greatest pathogen toxicity. The increased adherence and accumulation of AgNPs towards the membrane causes irreversible morphological changes, resulting in the loss of membrane integrity and permeability ${ }^{50}$. The use of surfaceengineered AgNPs may also provide a superior advantage to overcome biofilm recalcitrance to antimicrobials. For example, Xiaoning Li et al. show that the neutral and anionic particles cannot accumulate on the membrane or penetrate bacterial biofilms, while cationic particles readily penetrate and efficiently dissipate bacterial biofilms ${ }^{59}$. Collectively, it is expected that the interplay between AgNPs' size and surface structures will bring significant advancements for removing bacterial biofilms ${ }^{49}$. 
Role of silver and AgNPs in wound healing

Despite the positive anti-microbial effects of silver, negative effects on healthy tissues and cells have been observed ${ }^{28}$. However, these are dependent on several factors, including the mode of delivery, silver concentration, release rate, and duration of exposure ${ }^{23,37}$. A fine balance is therefore required between delivering an effective amount of silver for the elimination of bacteria that causes minimal skin damage ${ }^{54}$.

The negative effect of AgNPs is only observed when they are used at high concentrations or for prolonged exposures ${ }^{28}$. In contrast, silver nitrates, even at low concentrations, are shown to induce an excessive inflammatory response which can result in delayed wound healing ${ }^{60}$. Similarly, clinical studies have shown that continuous application of silver sulfadiazine Ag-SD can lead to hypertrophic scars and systemic and cutaneous argyria ${ }^{61,62}$. These side effects are due to the unregulated release of silver ions resulting in unnecessary tissue accumulation predisposing the host to tissue toxicity.

The widespread and prolonged use of silver dressings is still the subject of debate as the knowledge of their toxicological aspects emerges showing negative impacts on tissue integrity. The application of AgNPs could potentially overcome the current silver limitations. Tian et al. have shown that AgNPs can decrease inflammatory reactions by modulating cytokine levels and can facilitate the early phase of wound healing ${ }^{63}$. Similarly, Singla et al. saw faster wound healing with improved re-epithelisation, significant expression of collagen and growth factor, and improved vasculogenesis following treatment with $\mathrm{AgNPs}^{64}$.

Additionally, the role of AgNPs in promoting fibroblast migration and differentiation has been well documented; however, this remains contradictory ${ }^{65}$. For example, studies have shown that AgNPs are able to promote wound healing through positive effects on fibroblast and keratinocytes migration as well as the ability to modulate cell differentiation ${ }^{66,67}$. In contrast, other pre-clinical studies have shown that extracts of silver solution from commercial silver dressings induce a significant anti-proliferative effect on keratinocytes and fibroblasts ${ }^{68}$. In fact, topical applications of silver solutions have been shown to induce adverse effects on skin cell viability which restricts its widespread use ${ }^{69}$.

Nevertheless, silver in the form of NPs (AgNPs) offers numerous benefits beyond the antibacterial effect. In addition to its anti-inflammatory and proliferative effect, it has also been shown to down-regulate metalloproteinases (MMP) to an optimal level that facilitates faster healing ${ }^{70}$. However, despite the significant potential offered by AgNPs, emerging clinical studies have shown conflicting evidence; a recent international consensus document on the use of silver in wound care has highlighted that future clinical studies should focus on determining the underlying mechanisms of silver ions and wound healing responses ${ }^{5}$.

\section{Advanced therapeutic delivery of AgNPs}

Significant effort has been invested to develop a new generation of silver-based therapies focusing on those that can eradicate pathogenic bacteria with a minimal amount of silver for an extended period. Different types of dressings, coatings, nanofibres and hydrogels have been developed to maximise the therapeutic benefits of silver ${ }^{71-73}$. An ideal antibacterial, as shown in Figure 1, is expected to provide a moist wound environment, offer a protective role in secondary infections, remove wound exudate, and promote tissue regeneration ${ }^{74}$.

In particular, hydrogel, a three-dimensional porous structure with high swelling capacity, has proven to be the material of choice for topical wound application capable of stimulating the body's self-healing mechanism and promoting functional tissue development by providing a suitable microenvironment for healing ${ }^{75}$. Furthermore, hydrogels are biocompatible, have a consistency similar to the extracellular matrix (ECM), have the ability to remove excessive wound fluid, are biodegradable, and allow ease of synthesis ${ }^{76}$. In particular, the success of hydrogel delivery systems is highlighted by improved AgNPs stability, controlled drug release profile, and enhanced localised efficacy ${ }^{54}$. The hydrogel delivery system of AgNPs can be tailored for a prolonged delivery mode; this represents a great advantage concerning topical administration and sustained antibacterial efficacy. In addition, the physical and mechanical properties of the hydrogel are similar to the ECM, thereby presenting as an important supporting material for cellular proliferation and migration to stimulate key stages of wound healing ${ }^{77}$. Indeed, clinical studies to date have shown that the application of hydrogel biomaterials leads to improved wound healing outcomes $^{78,79}$.

Several studies have shown the possibility of incorporating AgNPs in various types of the hydrogel; these hydrogels are designed to either act as a 'smart' hydrogel $(\mathrm{pH}$ or temperature-responsive) or a simple polymeric carrier for improved delivery of therapeutics. In particular, smart hydrogels that trigger the release of AgNPs in response to environmental stimuli are gaining significant clinical attention for their potential to provide on-demand targeted delivery ${ }^{80}$. For example, Huang et al. have developed a multifunctional hydrogel encompassing both $\mathrm{pH}$ and temperature responsiveness properties while also providing extendedrelease of AgNPs for enhanced antibacterial effect ${ }^{81}$. Similarly, a recent in vitro study has shown controlled impregnation of ultrasmall AgNPs into a biocompatible thermo-responsive hydrogel for an extended-release profile with improved biofilm penetration capability ${ }^{54}$.

Additionally, other in vitro and in vivo studies have loaded AgNPs into different kinds of carriers, some of which include chitosan hydrogel to provide a synergistic antibacterial effect $^{82}$, polyvinyl alcohol/chitosan ${ }^{83}$, and injectable hydrogel systems ${ }^{84}$. In fact, current research is heavily focused on 
Figure 1. Illustration of an ideal antimicrobial wound dressing. The polymeric multipurpose hydrogel dressing is required to not only control bacterial infection but also promote tissue regeneration

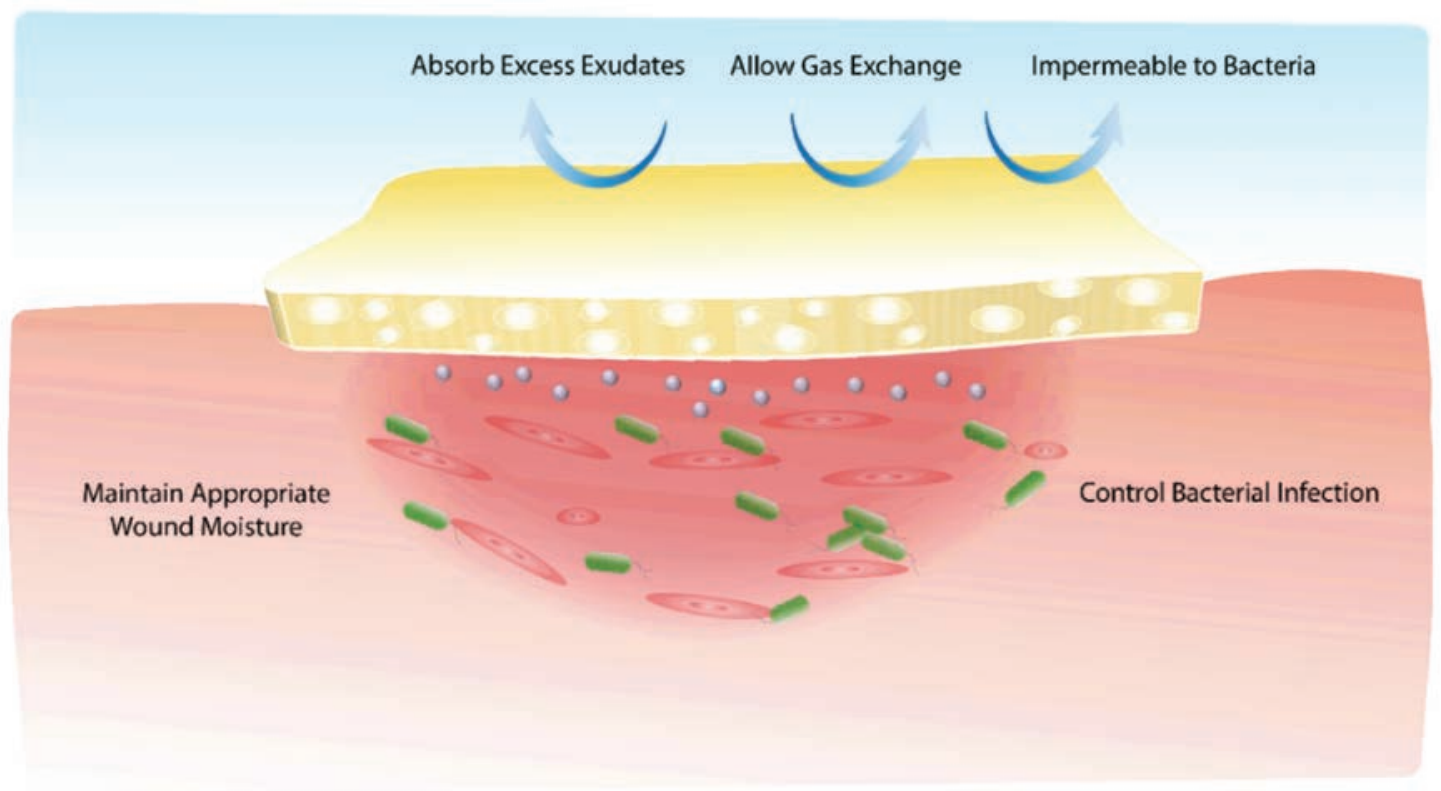

improved delivery of AgNPs using a variety of polymeric hydrogels either chemically or physically entrapped within the gel networks for slow and sustained release of silver, hence minimising the potentially toxic effects of silver ${ }^{2}$. In contrast to traditional silver dressings, AgNPs loaded into biocompatible hydrogels provide significant advantages through the capability to modulate the release properties and improve antibacterial effects. The release of AgNPs in a controlled and premeditated manner can be attained by modulating the hydrogel properties including porosity, diameters and degradability. As indicated by recent research, the advancement in hydrogel technology is expected to further accelerate to exponential levels to enable new functionalities and applicability in healthcare, paving the way for the development of an ideal AgNPs-loaded antimicrobial wound care product.

\section{Conclusion}

Despite significant advances in antimicrobial wound dressings, the treatment of infected wounds remains a significant clinical challenge. Recent progress has resulted in the development of numerous types of dressings, ranging from traditional to polymeric advanced dressings for different treatment purposes. However, no dressings are currently capable of providing multifunctional properties addressing all issues in chronic wounds, including controlling bacterial infection, regulating inflammation and promoting tissue regeneration. Medical devices and wound dressings impregnated with AgNPs may constitute an advanced and superior way to control acute and chronic bacterial wound infections. AgNPs present improvements on common antimicrobial strategies to overcome the current shortfalls due to bacterial resistance and improve therapeutic efficacy compared to conventional silver products (silver nitrate, silver sulfadiazine).
However, despite the recent progress, clinical approval of AgNP-based dressings is hampered by many challenging factors including the ability to control the silver loading, maintaining release profile, specificity to biological tissue, and biocompatibility. Therefore, it is anticipated that the safety of AgNPs-based formulations will be improved when encapsulated in biocompatible carriers such as hydrogels to combine multiple characteristics that are required to facilitate wound healing and infection control. AgNPs loaded into a stimuli-responsive hydrogel are gaining a lot of attention for their improved functionalities and capabilities for topical wound applications. Therefore, AgNPs-based hydrogels are expected to have great promise in treating acute and chronic wound infections.

\section{Conflict of interest}

The authors declare no conflicts of interest.

\section{Funding}

AJC is supported by the NHMRC Senior Research Fellowship (GNT \#1102617). ZK is supported by the University of South Australia Future Industries Institute Foundation Fellowship.

\section{References}

1. Eming SA, Martin P, Tomic-Canic M. Wound repair and regeneration: mechanisms, signaling, and translation. Sci Transl Med 2014;6(265):265sr6-265sr6.

2. Xu C, et al. Nanoparticle-based wound dressing: recent progress in the detection and therapy of bacterial infections. Bioconjug Chem 2020;31(7):1708-1723.

3. Percival SL, et al. A review of the scientific evidence for biofilms in wounds. Wound Repair Regen 2012;20(5):647-657.

4. Ogunniyi $A D$, et al. Bioluminescent murine models of bacterial sepsis and scald wound infections for antimicrobial efficacy testing. PLOS ONE 2018;13(7):e0200195. 
5. Bjarnsholt T, Edward-Jones V, Malone M, Ousey K, Rippon M, Rogers $A$, et al. The role of non-medicated dressings for the management of wound infection. 6th World Union of Wound Healing Societies. 10 Sep 2020 ed: Wounds International 2020. p. 1-32.

6. Malone M. Chapter 3: The microbiome of diabetic foot ulcers and the role of biofilms. In: Kon K, Rai M, editors. The microbiology of skin, soft tissue, bone and joint infections. Academic Press; 2017. p. 41-56.

7. Patel S. Understanding wound infection and colonisation. Wound Essentials. UK: Wounds International; 2007. p. 132-42.

8. Römling $U$, Balsalobre $C$. Biofilm infections, their resilience to therapy and innovative treatment strategies. J Intern Med 2012;272(6):541-561.

9. Woodward M. Silver dressings in wound healing: what is the evidence? Wound Pract \& Res 2005;13(4):153.

10. Flemming H.C, et al. Biofilms: an emergent form of bacterial life. Nat Rev Microbiol 2016;14(9):563-575.

11. Kopecki Z, Ogunniyi AD, Trott DJ, Cowin AJ. Fighting chronic wound infection - one model at a time. Wound Pract \& Res 2017;25(1):1-6.

12. Graves N, Zheng $\mathrm{H}$. Modelling the direct health care costs of chronic wounds in Australia. Wound Pract \& Res 2014;22(1):33.

13. Mitchell BG, et al. The burden of healthcare-associated infection in Australian hospitals: a systematic review of the literature. Infect, Dis \& Hlth 2017;22(3):117-128.

14. Malheiro J, Simões M. 4: Antimicrobial resistance of biofilms in medical devices. In: Deng Y, Lv W, editors. Biofilms and implantable medical devices. Woodhead Publishing; 2017. p. $97-113$.

15. Qayyum S, Khan AU. Nanoparticles vs. biofilms: a battle against another paradigm of antibiotic resistance. Med Chem Comm 2016;7(8):1479-1498.

16. Flemming H.-C, Wingender J. The biofilm matrix. Nat Rev Microbiol 2010;8(9):623-633.

17. Lansdown $A B$. A review of the use of silver in wound care: facts and fallacies. Br J Community Nurs 2004;13(Sup1):S6-S19.

18. Ayello EA, Carville K, Fletcher J, Keast D, Leaper D, Lindholm C, et al. Appropriate use of silver dressings in wounds. An expert working group consensus. London: Wounds International; 2012. p. 1-24.

19. Rai M, Gade A. Silver nanoparticles as a new generation of antimicrobials. Biotechnol Adv 2009;27(1):76-83.

20. Politano AD, et al. Use of silver in the prevention and treatment of infections: silver review. Surg Infect 2013;14(1):8-20.

21. Richter $\mathrm{K}$ et al. Taking the silver bullet colloidal silver particles for the topical treatment of biofilm-related infections. ACS Appl Mater Interfaces 2017;9(26):21631-21638.

22. Rigo $\mathrm{C}$, et al. Active silver nanoparticles for wound healing. Int $\mathrm{J}$ Mol Sci 2013;14(3):4817-40.

23. Kapp. H, Smola. H. Reduced cellular toxicity of a new silvercontaining antimicrobial dressing and its clinical performance in non-healing wounds. Wound Pract \& Res 2005;13(4):1-7.

24. Toy LW, Macera L. Evidence-based review of silver dressing use on chronic wounds. J Am Acad Nurse Pract 2011;23(4):183-92.

25. Docter $D$, et al. The nanoparticle biomolecule corona: lessons learned - challenge accepted? Chem Soc Rev 2015;44(17):60946121.

26. Le Ouay B, Stellacci F. Antibacterial activity of silver nanoparticles: a surface science insight. Nano Today 2015;10(3):339-354.

27. Atiyeh BS, et al. Effect of silver on burn wound infection control and healing: review of the literature. Burns 2007;33(2):139-148.
28. AshaRani PV, et al. Cytotoxicity and genotoxicity of silver nanoparticles in human cells. ACS Nano 2009;3(2):279-290.

29. Burd A, et al. A comparative study of the cytotoxicity of silverbased dressings in monolayer cell, tissue explant, and animal models. Wound Repair Regen 2007;15(1):94-104.

30. Hiro $\mathrm{ME}$, et al. Comparative evaluation of silver-containing antimicrobial dressings on in vitro and in vivo processes of wound healing. Eplasty 2012;12:e48-e48.

31. Lemire JA, Harrison JJ, Turner RJ. Antimicrobial activity of metals: mechanisms, molecular targets and applications. Nat Rev Microbiol 2013;11(6):371-384.

32. Tang S, Zheng J. Antibacterial activity of silver nanoparticles: structural effects. Adv Healthc Mater 2018;7(13):1701503.

33. Sim W, et al. Antimicrobial silver in medicinal and consumer applications: a patent review of the past decade (2007-2017). Antibiotics (Basel, Switzerland) 2018;7(4):93.

34. Miller KP, et al. Inorganic nanoparticles engineered to attack bacteria. Chem Soc Rev 2015;44(21):7787-7807.

35. Soares S, et al. Nanomedicine: principles, properties, and regulatory issues. Front Chem 2018;6(360).

36. Makvandi $P$, et al. Metal-based nanomaterials in biomedical applications: antimicrobial activity and cytotoxicity aspects. Adv Funct Mater 2020;30(22):1910021.

37. Medici S, et al. Medical uses of silver: history, myths, and scientific evidence. J Med Chem 2019;62(13):5923-5943.

38. Jeelani PG, et al. Multifaceted application of silica nanoparticles: a review. Silicon 2020;12(6):1337-1354.

39. De Jong WH, Borm PJA. Drug delivery and nanoparticles: applications and hazards. Int J Nanomedicine 2008;3(2):133149.

40. Beer C, et al. Toxicity of silver nanoparticles: nanoparticle or silver ion? Toxicol Lett 2012;208(3):286-292.

41. Foroozandeh P, Aziz AA. Insight into cellular uptake and intracellular trafficking of nanoparticles. Nanoscale Res Lett 2018;13(1):339-339.

42. Ivask $A$, et al. Toxicity mechanisms in Escherichia coli vary for silver nanoparticles and differ from ionic silver. ACS Nano 2014;8(1):374-386.

43. Hwang ET, et al. Analysis of the toxic mode of action of silver nanoparticles using stress-specific bioluminescent bacteria. Small 2008;4(6):746-750.

44. Rinna A, et al. Effect of silver nanoparticles on mitogen-activated protein kinases activation: role of reactive oxygen species and implication in DNA damage. Mutagenesis 2014;30(1):59-66.

45. Kim JS, et al. Antimicrobial effects of silver nanoparticles. Nanomed 2007;3(1):95-101.

46. Mahmoudi M, Serpooshan V. Silver-coated engineered magnetic nanoparticles are promising for the success in the fight against antibacterial resistance threat. ACS Nano 2012;6(3):2656-2664.

47. McShan D, Ray PC, Yu H. Molecular toxicity mechanism of nanosilver. J Food Drug Anal 2014;22(1):116-127.

48. Nel AE, et al. Understanding biophysicochemical interactions at the nano-bio interface. Nat Mater 2009;8(7):543-557.

49. Haidari $\mathrm{H}$, et al. The interplay between size and valence state on the antibacterial activity of sub-10nm silver nanoparticles. Nanoscale Adv 2019;1(6):2365-2371.

50. Jose Ruben M, et al. The bactericidal effect of silver nanoparticles. Nanotechnol 2005;16(10):2346-2353.

51. Agnihotri S, Mukherji S, Mukherji S. Size-controlled silver nanoparticles synthesized over the range 5-100nm using the same protocol and their antibacterial efficacy. RSC Adv 2014;4(8):3974-3983. 
52. Yuan $\mathrm{X}$, et al. Ultrasmall Ag+-rich nanoclusters as highly efficient nanoreservoirs for bacterial killing. Nano Res 2014;7(3):301-307.

53. Peulen T.-O, Wilkinson KJ. Diffusion of nanoparticles in a biofilm. Environ Sci Technol 2011;45(8):3367-3373.

54. Haidari $\mathrm{H}$, et al. Ultrasmall AgNP-impregnated biocompatible hydrogel with highly effective biofilm elimination properties. ACS Appl Mater Interfaces 2020;12(37):41011-41025.

55. Choi O, Hu Z. Size dependent and reactive oxygen species related nanosilver toxicity to nitrifying bacteria. Environ Sci Technol 2008;42(12):4583-4588.

56. Liu Y, et al. Surface-adaptive, antimicrobially loaded, micellar nanocarriers with enhanced penetration and killing efficiency in Staphylococcal biofilms. ACS Nano 2016;10(4):4779-4789.

57. Abbaszadegan $A$, et al. The effect of charge at the surface of silver nanoparticles on antimicrobial activity against grampositive and gram-negative bacteria: a preliminary study. $\mathrm{J}$ Nanomater 2015;720654.

58. El Badawy AM, et al. Surface charge-dependent toxicity of silver nanoparticles. Environ Sci Technol 2011;45(1):283-287.

59. Li X, et al. Control of nanoparticle penetration into biofilms through surface design. Chem Commun 2015;51(2):282-285.

60. Warriner R, Burrell R. Infection and the chronic wound: a focus on silver. Adv Skin Wound Care 2005;18(8):2-12.

61. Fisher NM, Marsh E, Lazova R. Scar-localized argyria secondary to silver sulfadiazine cream. J Am Acad Dermatol 2003;49(4):730732.

62. Heyneman A, et al. The role of silver sulphadiazine in the conservative treatment of partial thickness burn wounds: a systematic review. Burns 2016;42(7):1377-1386.

63. Tian J, et al. Topical delivery of silver nanoparticles promotes wound healing. Chem Med Chem 2007;2(1):129-136.

64. Singla $R$, et al. In vivo diabetic wound healing potential of nanobiocomposites containing bamboo cellulose nanocrystals impregnated with silver nanoparticles. Int $\mathrm{J}$ Biol Macromol 2017; 105:45-55.

65. Frankova $\mathrm{J}$, et al. Effects of silver nanoparticles on primary cell cultures of fibroblasts and keratinocytes in a wound-healing model. J Appl Biomater Funct Mater 2016;14(2):e137-42.

66. You C, et al. Silver nanoparticle loaded collagen/chitosan scaffolds promote wound healing via regulating fibroblast migration and macrophage activation. Sci Rep 2017;7(1):1048910489.

67. Liu X, et al. Silver nanoparticles mediate differential responses in keratinocytes and fibroblasts during skin wound healing. Chem Med Chem 2010;5(3):468-75.

68. Paddle-Ledinek JE, Nasa Z, Cleland HJ. Effect of different wound dressings on cell viability and proliferation. Plast Reconstr Surg 2006;117(7 Suppl):110S-118S; discussion 119S-120S.

69. Poon VKM, Burd A. In vitro cytotoxity of silver: implication for clinical wound care. Burns 2004;30(2):140-147.

70. Ahsan A, Farooq MA. Therapeutic potential of green synthesized silver nanoparticles loaded PVA hydrogel patches for wound healing. J Drug Deliv Sci Technol 2019;54:101308.

71. Vasilev K, et al. Tunable antibacterial coatings that support mammalian cell growth. Nano Lett 2010;10(1):202-207.

72. Taheri S, et al. Substrate independent silver nanoparticle based antibacterial coatings. Biomaterials 2014;35(16):4601-4609.

73. Ravindran Girija $A$, et al. Ultrasmall gold nanocluster based antibacterial nanoaggregates for infectious wound healing. Chem Nano Mat 2019;5(9):1176-1181.

74. Haidari $\mathrm{H}$, et al. Development of topical delivery systems for flightless neutralizing antibody. J Pharm Sci 2017;106(7):17951804.
75. Gupta P, Vermani K, Garg S. Hydrogels: from controlled release to pH-responsive drug delivery. Drug Discov Today 2002;7(10):569579.

76. Arafa MG, El-Kased RF, Elmazar MM. Thermoresponsive gels containing gold nanoparticles as smart antibacterial and wound healing agents. Sci Rep 2018;8(1):13674.

77. Liu $\mathrm{H}$, et al. A functional chitosan-based hydrogel as a wound dressing and drug delivery system in the treatment of wound healing. RSC Adv 2018;8(14):7533-7549.

78. Tran NQ, et al. In situ forming and rutin-releasing chitosan hydrogels as injectable dressings for dermal wound healing. Biomacromol 2011;12(8):2872-2880.

79. Chen $\mathrm{G}$, et al. Bioinspired multifunctional hybrid hydrogel promotes wound healing. Adv Funct Mater 2018;28(33):1801386.

80. Cavallaro A, Taheri S, Vasilev K. Responsive and "smart" antibacterial surfaces: common approaches and new developments (Review). Biointerphas 2014;9(2):029005.

81. Huang W.-C, etal. A macroporous hydrogeldressing with enhanced antibacterial and anti-inflammatory capabilities for accelerated wound healing. Adv Funct Mater 2020;30(21):2000644.

82. Huang $X$, et al. A novel silver-loaded chitosan composite sponge with sustained silver release as a long-lasting antimicrobial dressing. RSC Adv 2017;7(55):34655-34663.

83. Yang W, et al. Polyvinyl alcohol/chitosan hydrogels with enhanced antioxidant and antibacterial properties induced by lignin nanoparticles. Carbohydr Polym 2018;181:275-284.

84. Li L, et al. Injectable self-healing hydrogel with antimicrobial and antifouling properties. ACS Appl Mater Interfaces 2017;9(11):9221-9225. 